ECCOMAS

Proceedia
COMPDYN 2021

$8^{\text {th }}$ ECCOMAS Thematic Conference on Computational Methods in Structural Dynamics and Earthquake Engineering M. Papadrakakis, M. Fragiadakis (eds.) Streamed from Athens, Greece, 28 - 30 June 2021

\title{
SIMULATING LOW FREQUENCY SOUND TRANSMISSION LOSS OF MOUNTED PANELS
}

\author{
Moris Kalderon ${ }^{1}$, Andreas Paradeisiotis ${ }^{1}$ and Ioannis Antoniadis ${ }^{1}$ \\ ${ }^{1}$ National Technical University of Athens Dynamics and Structures Laboratory, School of Mechanical \\ Engineering \\ Heroon Polytechniou 9, 15780 Zografou Athens, Greece \\ e-mail: moriska@mail.ntua.gr, aparadis@mail.ntua.gr
}

\begin{abstract}
Lightweight panels are very common in the building, aerospace and automotive industry, and their acoustic insulation properties are well studied; nevertheless, low frequency noise is inherently difficult to deal with, mainly due to its high penetration. To encounter this issue, several treatments have been proposed in the form of perforated plates, meta-surfaces and acoustic/elastic meta-materials among others. Despite, the continuous attempts, none of the available solutions has indicated an outstanding performance in the low frequency range. On the other hand, boundary conditions of a vibrating panel are known to have an influence on the sound transmission loss (STL). Driven by the recently extended standards to include frequencies as low as $50 \mathrm{~Hz}$, and the academic community debate about the reliability of lowfrequency measurements in reverberant chambers, STL improvement in the region of the first panel resonance is investigated in this paper, considering a panel supported on elastic mounts. Following a general vibration isolation approach, the fundamental eigenfrequency of the panelmount system is reduced and is explicitly selected below the threshold of human hearing. STL predictions are accomplished utilizing a Finite Element (FE) vibroacoustic model that simulates real test room conditions. Compared to the commonly used models of a baffled plate radiating to infinity, this detailed approach allows not only a more accurate calculation of STL but also reveals the potential issues emerging during laboratory measurements.
\end{abstract}

Keywords: low frequency, panel, sound transmission loss, elastic mounts, reverberation room

ISSN:2623-3347 (C) 2021 The Authors. Published by Eccomas Proceedia.

Peer-review under responsibility of the organizing committee of COMPDYN 2021.

doi: $10.7712 / 120121.8861 .18701$ 


\section{INTRODUCTION}

When considering room-to-room noise transmission inside buildings, sources such as electrical appliances, music and movies in neighboring apartments etc., have notable low frequency content. Another significant component of low frequency noise in residential areas is road noise emitted by vehicles, since their engines operate typically at speeds up to 6000 RPM (100 $\mathrm{Hz}$ ). Therefore, there is an ever increasing demand for noise mitigation solutions in this range, providing an improvement on the residents quality of life but also for compliance with legal requirements [1].

However, the low frequency range approximately below $200 \mathrm{~Hz}$, has been overwhelmingly overlooked in the past when considering applications and we presently lack understanding to properly model the processes governing the sound transfer in this frequency range. Almost exclusively, the focus is on the region of the coincidence frequency of finite partitions. The lack of complete industrial standardization and guidances for such cases, especially below $100 \mathrm{~Hz}$ is also a factor. Yet, a large research program on low frequency sound transmission into buildings was conducted by NGI (the Norwegian Geotechnical Institute) between 2010-2016, comprising of laboratory, full scale and numerical testing [2]. The disseminating research findings of the program have been proven very useful, but there are still uncharted areas in this field.

When it comes to acoustic treatment in rooms there are also a lot of misconceptions among the general public. Full scale tests reveal that when noise transmission occurs inside a building, the peaks of the low frequency sound spectrum are of the same order as the fundamental acoustic room-modes [3], leading to a collection of resonances, which compromise the measurements. International standards describe procedures to extend the measurement range down to the 50 $\mathrm{Hz}$ one-third octave band $[4,5,6]$. Prescriptions include use of specific source and receiver positions, however the procedure is complex, with the worst case scenario to propose the use of a sufficiently large number of source-receiver combinations as a means to smooth the large spatial variations in measured levels. Moreover, regarding the laboratory geometry, large rooms with volumes $V>200 \mathrm{~m}^{3}$ and specific room size ratios are suggested when low frequency measurements are conducted. Recently, Ayr et al. [7] presented a detailed low frequency qualification procedure for a typical reverberant test room in order to perform sound power measurements, by taking advantage of a room finite element model. Similarly, Løvholt et al. [8] developed a finite element methodology that incorporates a two-way coupled fluid-structure interaction and they compared their numerical results with experimental measurements including low frequency sound transmission of plain walls, and walls with windows. Vorländer and Aretz also worked on room to room acoustic simulations, focusing on the room boundary conditions [9] and the uncertainties of the computational models [10].

As far as the analytic methods are concerned, the acoustic radiation from plates has long been an important subject in structural dynamics and acoustics, thus numerous mathematical models describing the structural response to noise, acoustic fatigue and sound transmission of partitions have been developed. Beranek and Ver, Cremer et al., Ordubadi and Lyon and London $[11,12,13,14]$ derived equations for the unbounded/infinite partitions ("infinite-panel theory") simplifying the problem and later ad hoc corrections were added to enhance the accuracy of the Sound Transmission Loss (STL) formulas. Although, the infinite panel theory can approximate pretty accurately the panel response when high frequency noise mitigation is concerned, it is known that bounded plates vibrating at frequencies below the critical frequency are very much influenced by the presence of their boundaries [15, 16]. Similarly, Kim [17] approached sound transmission through partitions from the standpoint of impedance mismatch. Despite, the merits 
of the aforementioned approaches, in low frequency the modal response of the structure affects the transmission properties.

The aim of this contribution is to investigate the effects of elastic mounting in the STL performance, considering a much relevant specific case of single-leaf plasterboard partitions and show how the uncertainties due to practical factors can be diminished with this approach. Namely, the ways that elastic mounting may be incorporated in already existing panels and set circumstances regarding their spatial configuration, as well as, the effectiveness of the damping of the mounts in the mitigation of the first panel resonance. The analysis is conducted via both simple mathematical models and a coupled finite element vibroacoustic model where the room to room sound transmission is described based on the laboratory set-up in SINTEF Building laboratory in Oslo, Norway [8].

The idea of utilizing "soft mounts" to reduce the fundamental frequency of the system delves into the investigation of what constitutes soft mounting. Therefore, a modeling procedure based on certain approximations [18] is formulated in order to provide a projection of the expected level of stiffness that satisfies this terminology and serves the intended purpose. Intuitively, following the general concepts of vibration isolation for the reduction of the eigenfrequency through the reduction of stiffness, supporting an existing panel on elastic mounts, the 'mass law' behavior may be dragged into lower frequencies and the resonant region of the new system panel-mount will be moved below a critical frequency, for example at $20 \mathrm{~Hz}$ considering the threshold of human hearing.

To sum up, in Section 2 the theoretical formulation of the three applied methods, are provided together with the FE model describing the room to room transmission. In Section 3 a parametric investigation is presented to reveal the parameters that affect STL of a rectangular panel and finally in Section 4 the conclusions of this work are briefly discussed.

\section{MATERIALS \& METHODS}

By defining the transmission coefficient as

$$
\tau=\frac{\Pi_{t}}{\Pi_{i}}
$$

where $\Pi_{i}$ is the incident and $\Pi_{t}$ the transmitted (radiated) sound power, the STL is expressed as

$$
S T L=10 \log _{10}\left(\frac{1}{\tau}\right)
$$

A typical form of the STL curve for sound propagation through a thin panel is decribed in [19]. In the 'mass law' region, the STL is controlled by the mass per unit area of the panel. Specifically, for a thin panel, neglecting stiffness and damping (limp wall):

$$
S T L=10 \log _{10}\left[\frac{\Omega^{2} \bar{m}^{2}}{4\left(\rho_{0} c_{0}\right)^{2} / \cos ^{2} \theta_{i}}\right]
$$

where $\Omega$ is the cyclical frequency of the propagated incident sound waves, $\theta_{i}$ is the angle of incidence, $c_{0}$ is the speed of sound in air, $\rho_{0}$ is the air density and $\bar{m}\left[\mathrm{~kg} / \mathrm{m}^{2}\right]$ is the mass per unit area of the panel. Although the mass law is very simple and compact, it is only valid for large simple plates because it is derived from the infinite and rigid plate assumption. Therefore, attention should be given when utilized for STL predictions of general finite panels. 


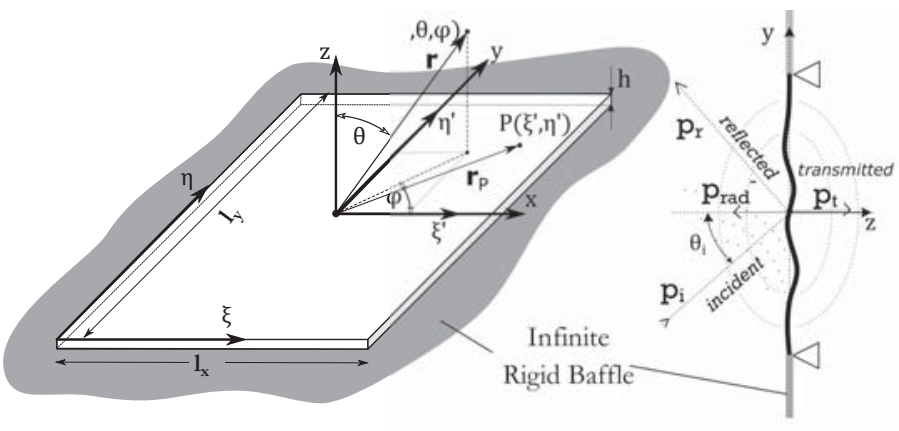

(a)

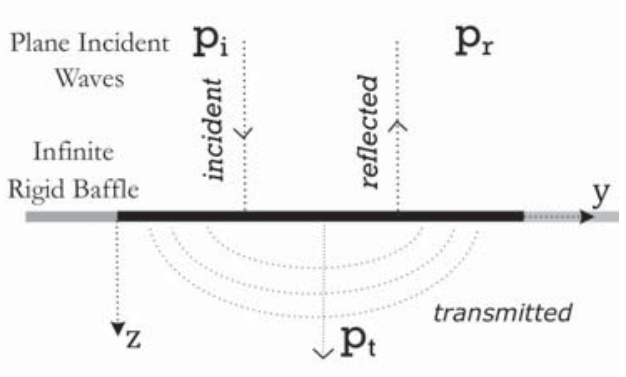

(b)

Figure 1: $(\alpha)$ Coordinate systems and transmission geometry of a rectangular plate in a rigid baffle. (b) Transmission geometry of the free finite rigid panel.

\subsection{Sound radiation from a rectangular panel in an infinite rigid baffle}

The fluid domain is assumed homogeneous and compressible. The mass density of air and sound speed are noted as $\rho_{0}$ and $c_{0}$, respectively. The infinitely large acoustic rigid baffle divides the space into two domains i.e. the excitation domain $V^{-}$and the receiver domain $V^{+}$. The panel is subjected to the incident pressure $p_{i}$, with incidence angle $\theta_{i}$ and azimuth angle $\phi_{i}$. Therefore, the excitation domain consists in the incident pressure $p_{i}$, the reflected pressure $p_{r}$, and the re-radiated pressure $p_{\text {rad }}^{-}$due to the motion of the finite panel. The receiver domain consists only in the transmitted pressure $p_{t}$, namely the radiated pressure due to the motion of the panel $p_{\text {rad }}^{+}$. Assuming that the incident sound pressure $p_{i}$ is a harmonic function, we can express it as

$$
p_{i}(x, y, z, t)=P_{i} e^{-\jmath\left(\kappa_{x} x+\kappa_{y} y+\kappa_{z} z\right)} e^{\jmath \Omega t}=\tilde{p}_{i}(x, y, z) e^{\jmath \Omega t}
$$

where $\tilde{p}_{i}(x, y, z)$ the time invariable complex amplitude and $\kappa=\Omega / c_{0}$ is the wavenumber with

$$
\kappa_{x}=\kappa \sin \theta \cos \phi \quad, \quad \kappa_{y}=\kappa \sin \theta \sin \phi \quad, \quad \kappa_{z}=\kappa \cos \theta
$$

The reflected pressure by the surface of the panel is then expressed as

$$
p_{r}(x, y, z, t)=P_{r} e^{-\jmath\left(\kappa_{x} x+\kappa_{y} y-\kappa_{z} z\right)} e^{\jmath \Omega t}=\tilde{p}_{r}(x, y, z) e^{\jmath \Omega t}
$$

denoting the opposite direction from the incident wave. Lord Rayleigh [20] determined a relationship expressing the radiated (transmitted) pressure in terms of the structural velocity based on Green's function,

$$
p_{\text {rad }}^{+}(\mathbf{r}, t)=p_{t}(\mathbf{r}, t)=\frac{\jmath \Omega \rho_{0}}{2 \pi} e^{\jmath \Omega t} \int_{-l_{x} / 2}^{l_{x} / 2} \int_{-l_{y} / 2}^{l_{y} / 2} \frac{e^{-\jmath \kappa R}}{R} \frac{\partial w\left(\xi^{\prime}, \eta^{\prime}\right)}{\partial t} d \eta^{\prime} d \xi^{\prime}
$$

where $R$ referring to Fig.1, is defined as

$$
R=\left|\mathbf{r}-\mathbf{r}_{\mathbf{P}}\right|=\sqrt{\left(x-\xi^{\prime}\right)^{2}+\left(y-\eta^{\prime}\right)^{2}+z^{2}}
$$

and $\mathbf{r}=(x, y, z)$ is the position vector of a point in the receiver domain while $\mathbf{r}_{P}=\left(\xi^{\prime}, \eta^{\prime}, 0\right)$ is the position vector of the center of an elemental radiator with surface $\delta S$ on the panel, having a normal velocity amplitude $\dot{w}\left(\xi^{\prime}, \eta^{\prime}\right)$. For the local coordinates holds that $\xi^{\prime}=\xi-\frac{l_{x}}{2}, \eta^{\prime}=\eta-\frac{l_{y}}{2}$.

Following the calculation of the expression for the transmitted pressure, there are two different ways used to determine the radiated (transmitted) power. The first is to integrate the 
intensity on a hemisphere in the far-field enclosing the plate which is followed in this paper, and the latter is to integrate the acoustic intensity over the surface of the vibrating plate. Both approaches require the knowledge of the distribution of the velocity over the plate and they assume a weak coupling between the vibrating structure and the radiated sound field.

Applying the far-field approximation as described in Section A.1, the transmitted intensity $I_{t}$ is calculated as

$$
I_{t}(r, \theta, \phi)=\frac{\left|p_{t}(r, \theta, \phi)\right|^{2}}{2 \rho_{0} c_{0}}
$$

Then, the transmitted power $\Pi_{t}$ comes by integrating the transmitted intensity on a hemisphere in the far field enclosing the plate as

$$
\Pi_{t}=\int_{0}^{2 \pi} \int_{0}^{\pi / 2} I_{t} r^{2} \sin \theta d \theta d \phi
$$

while the incident power on the panel is defined as

$$
\Pi_{i}=\frac{\left|p_{i}\right|^{2} l_{x} l_{y} \cos \theta}{2 \rho_{0} c_{0}}
$$

\subsection{Vibration of a panel subject to external pressure}

With reference to Fig. $1 \alpha$, the finite-sized flat panel partition is assumed to be rectangular and baffled, with lengths $l_{x}$ and $l_{y}$ along the $x$ and $y$ axes respectively. The panel of thickness $h$, is considered homogeneous and isotropic and is modeled as a classical thin plate, implying that the effects of both the rotary inertia and the transverse shear deformation can be neglected. Hereinafter, the equation of motion governing the bending vibration of the plate is given by

$$
\begin{aligned}
D\left[\frac{\partial^{4} w(\xi, \eta, t)}{\partial \xi^{4}}\right. & \left.+2 \frac{\partial^{4} w(\xi, \eta, t)}{\partial \xi^{2} \partial \eta^{2}}+\frac{\partial^{4} w(\xi, \eta, t)}{\partial \eta^{4}}\right]+\rho h \frac{\partial^{2} w(\xi, \eta, t)}{\partial t^{2}}= \\
& =p_{i}(\xi, \eta, t)+p_{r}(\xi, \eta, t)+p_{\text {rad }}^{-}-p_{\text {rad }}^{+}
\end{aligned}
$$

where $D=\tilde{E} h^{3} / 12\left(1-\nu^{2}\right)$ is the bending stiffness of the plate, $E, h, \rho$ and $\nu$ are the Young's modulus, thickness, mass density and Poisson's ratio of the plate, respectively. $w(\xi, \eta, t)$ is the instantaneous transverse displacement. In order to account for energy dissipation due to structural damping, a complex modulus of elasticity is introduced $\tilde{E}=E(1+j n)$, where $n$ is the loss factor.

The right hand side must satisfy the velocity continuity at the surface of the panel $(z=0)$. In that case, it holds that $p_{r}=p_{i}$ and also since $p_{t}=p_{\text {rad }}^{+}=-p_{\text {rad }}^{-}$the right hand side becomes

$$
p_{i}(\xi, \eta, t)+p_{r}(\xi, \eta, t)+p_{\text {rad }}^{-}-p_{\text {rad }}^{+}=2 p_{i}(\xi, \eta, t)-2 p_{t}(\xi, \eta, t)
$$

The so-called "blocked pressure" [17] $p_{b}=2 p_{i}$, is the pressure when the incident wave meets a rigid wall.

\subsection{Free Finite Rigid (FFR) Panel Approximation}

In order to replace the infinite panel approximation (limp wall) with a more appropriate reference for finite panels, the free finite rigid (FFR) panel approximation is formulated. In this case it is assumed that the panel is rigid, consisting only in mass and oscillating freely in an infinite rigid baffle but instead has finite dimensions as demonstrated in Fig.1b. 
Since the panel is considered rigid, the spatial derivatives of Eq.(12) are eliminated. Also, considering only the blocked pressure $p_{b}=2 p_{i}$ as the forcing pressure and that $p_{i}$ are incident plane waves, the equation of motion becomes:

$$
\rho h \ddot{w}=2 P_{i} e^{\jmath \Omega t}
$$

The forcing pressure is a harmonic function, therefore the steady-state transverse displacement of the panel can be expressed as

$$
w=W(\xi, \eta) q(t)
$$

where

$$
q(t)=C e^{\jmath \Omega t}
$$

For the rigid assumption, the shape function is independent of the position $(\xi, \eta)$ on the surface of the panel, namely $W(\xi, \eta)=1$. Substituting the solution into Eq.(14) and solving for $C$ gives:

$$
C_{\mathrm{FFR}}=-\frac{2 P_{i}}{\rho h \Omega^{2}}
$$

The transmission coefficient then comes as

$$
\tau_{\mathrm{FFR}}=\frac{1}{l_{x} l_{y}}\left(\frac{\rho_{0}}{\pi}\right)^{2} \frac{1}{\bar{m}^{2}} I_{\theta \phi}
$$

where $\bar{m}=\rho h$ is the mass density (mass per surface area) of the panel, and $I_{\theta \phi}$ is the double integral over a hemisphere in the receiver domain as given in Eq.(A.10).

\subsection{Simply Supported Plate First Mode Approximation}

Analytic solutions for the problem can be found via modal superposition methods, for example as formulated by Roussos [21]. However, in these analytic approaches, past researchers usually avoided to include in their calculations the re-radiated pressure $2 p_{t}(\xi, \eta, t)$ (often referred to as "fluid loading") of Eq.(13) in order to reduce complexity. According to Roussos [21] neglecting the effect of the re-radiated pressure allows an accurate solution to be obtained over large frequency range, and gives invalid answers only for frequencies near the panel's fundamental resonant frequency.

Since the low frequency range of interest in the present case is exactly the range near the fundamental resonance of the panel, an attempt for a more accurate and usable approximation is formulated in this section, including the effect of fluid loading.

Considering only the $1^{\text {st }}$ vibrational mode of the simply supported plate, the spatially dependent amplitude of the transverse displacement $w(\xi, \eta)=W_{1}(\xi, \eta, t) e^{-\jmath \Omega t}$ is assumed by the shape function

$$
W_{1}=C_{1} \sin \left(\frac{\pi \xi}{l_{x}}\right) \sin \left(\frac{\pi \eta}{l_{y}}\right)
$$

the resulting expression for the transmitted (radiated) power $\Pi_{t}$ is given by Eq.(A.25). The detailed analysis is presented in Section A.3.

Assuming normal incident waves $\left(\theta_{i}=0\right)$, the incident power is given by

$$
\Pi_{i}=\frac{P_{i}^{2} l_{x} l_{y}}{2 \rho_{0} c_{0}}
$$


leading to the transmission coefficient $\tau$ as

$$
\tau_{1}=\frac{1}{l_{x} l_{y}} \frac{\left(16 \Omega^{2} \rho_{0}\right)^{2}}{\left|\pi^{3} \rho h\left(\omega_{1}^{2}-\Omega^{2}\right)-16 \Omega^{2} \rho_{0} \frac{e^{-j k r}}{r}\right|^{2}} I_{\theta \phi}
$$

where distance $r$ needs to be large enough to satisfy the far-field approximation and $\omega_{1}$ is the natural frequency of the $1^{\text {st }}$ mode resulting from the homogeneous form of Eq. (12), as

$$
\omega_{1}^{2}=\frac{D \pi^{4}}{\rho h}\left(\frac{1}{l_{x}^{2}}+\frac{1}{l_{y}^{2}}\right)^{2}
$$

Expressing the dynamic behavior of the continuous system of the simply supported panel approximated by the $1^{\text {st }}$ mode, as a single degree of freedom (SDoF) lumped parameter model (LPM), the corresponding generalized mass and stiffness [22] come as

$$
\begin{aligned}
m_{1}^{*} & =\frac{\rho h l_{x} l_{y}}{4}=\frac{\bar{m} l_{x} l_{y}}{4}=\frac{m}{4} \\
k_{1}^{*} & =\frac{D \pi^{4} l_{x} l_{y}}{4}\left(\frac{1}{l_{x}^{2}}+\frac{1}{l_{y}^{2}}\right)^{2}
\end{aligned}
$$

The transmission coefficient is then calculated as

$$
\tau_{\mathrm{LPM}}=l_{x} l_{y}\left(\frac{4 \Omega^{2} \rho_{0}}{\pi^{3}}\right)^{2} \frac{1}{\left[m_{1}^{*}\left|\omega_{1}^{2}-\Omega^{2}\right|\right]^{2}} I_{\theta \phi}
$$

\subsection{Lamped parameter model for elastic mounts}

The goal is to formulate a straightforward procedure for the investigation of how the bending stiffness of the panel combines with the mounting stiffness in the case where the panel is supported on elastic mounts and how the acoustic performance is affected. In order to do that, the main assumption is that the bending stiffness of the deformable plate is in a way in series with the stiffness of the mounting. Therefore, the resulting stiffness is

$$
k_{t o t}=\frac{k_{1}^{*} k_{0}^{\prime}}{k_{1}^{*}+k_{0}^{\prime}}
$$

When the mounting is very stiff $\left(k_{0}^{\prime}>>k_{1}^{*}\right)$ leads to $k_{t o t}=k_{1}^{*}$, namely, the case of the simply supported plate is approached. When $k_{0}^{\prime} \rightarrow 0$, the plate is essentially free floating. Naturally, when the eigenfrequency of the system is reduced along with the total stiffness, the mass considered in the SDoF model increases. In this case this variation is neglected for reasons of simplification. As a result, when the considered mounting stiffness $k_{0}^{\prime}$ is of comparative order of magnitude with $k_{1}^{*}$, there is some deviation between the resulting eigenfrequency of the model and the actual eigenfrequency. However, in cases when $k_{0}^{\prime}>>k_{1}^{*}$ or $k_{0}^{\prime}<<k_{1} *$ the deviation is nullified. Thus the model is used for an estimation of the required stiffness level and guidance for the design of the mounts.

Fig.2, shows the equivalent dynamic SDOF model of the deformable thin panel when it is supported on elastic mounts according to the 'in-series' assumption. This is achieved by utilizing the generalized values of the structure, namely generalized mass and the corresponding 


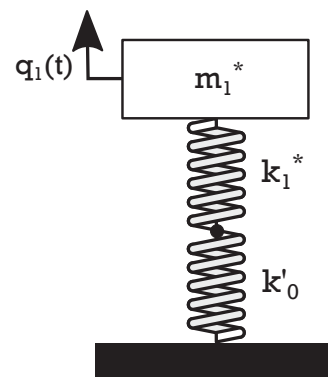

Figure 2: Modelling of a deformable plate on elastic mounts with stiffness $k_{0}^{\prime}$.

generalized stiffness for the appropriate modes, as calculated from Eqs.(23),(24) for the $1_{s t}$ mode approximation of the simply supported panel.

Since the loss factor $(n)$ can represent more accurately the dynamic response of nonlinear systems compared with the damping ratio which is defined on the grounds of the linear single degree of freedom (SDOF) viscous model [23, 24], hysteretic damping is introduced indirectly considering complex stiffness elements as

$$
k_{0}^{\prime}=k_{0}(1+\jmath n)
$$

The STL of the model is then calculated as $S T L=10 \log _{10}\left(\frac{1}{\tau}\right)$, where the transmission coefficient $\tau$ comes as

$$
\tau=l_{x} l_{y}\left(\frac{4 \Omega^{2} \rho_{0}}{\pi^{3}}\right)^{2} \frac{1}{\left.\mid-\Omega^{2} m_{1}^{*}+k_{t o t}\right)\left.\right|^{2}} I_{\theta \phi}
$$

\subsection{D vibro-acoustic FE model}

In addition to the aforementioned analytical methodologies, a 3-D FE coupled vibro-acoustic model was developed on the commercial finite element software package ABAQUS [25]. In the present simulations, the geometry of the SINTEF laboratory [8] is utilized as the computational domain, as shown in Fig. $3 \alpha$ and b. It is noted that the laboratory geometry and the measurement procedure do not follow accurately the proposed from the standards guidance, however, the developed model can be used for room to room sound transmission predictions. The panel is excited by the source located in the left corner of the source room and the incidence and radiated acoustic pressure is extracted from the fluid-structure interface nodes. An arithmetic average is performed by

$$
\begin{aligned}
& \overline{P_{t}}=\frac{1}{n} \sum_{i=1}^{n}\left|p_{t, i}\right| \\
& \overline{P_{i}}=\frac{1}{n} \sum_{i=1}^{n}\left|p_{i, i}\right|
\end{aligned}
$$

where $n$ denotes the total number of measuring nodes; $i$ denotes the node number; $p_{i}$ denotes the incidence pressure and $p_{t}$ denotes the transmitted pressure. Then the averaged pressures $\overline{P_{i}}, \overline{P_{t}}$ are used to compute the simulated STL by

$$
S T L=20 \log _{10} \frac{\left|\overline{P_{i}}\right|}{\overline{P_{t}}}
$$




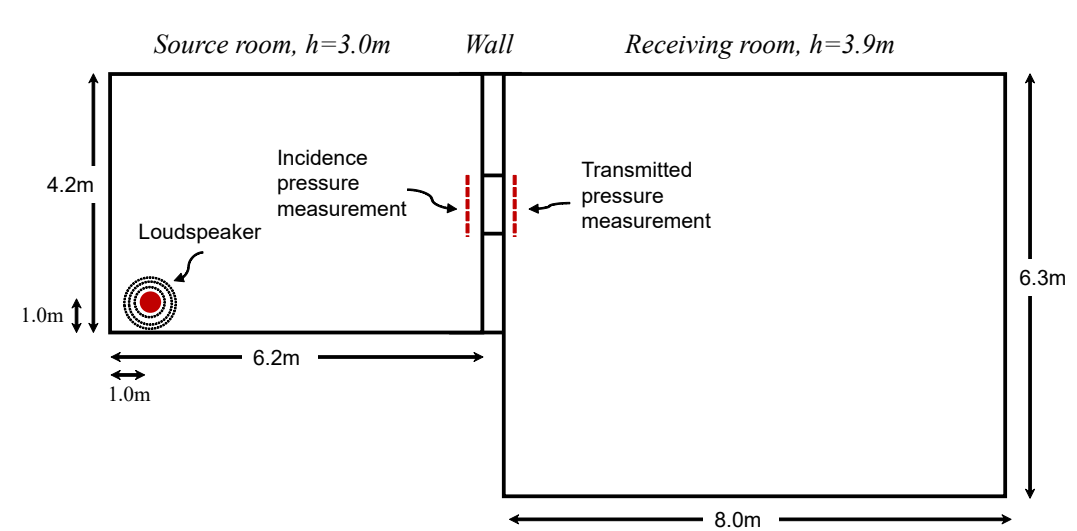

(a)

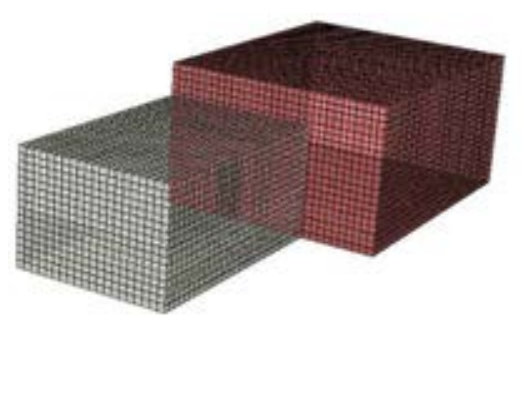

(b)

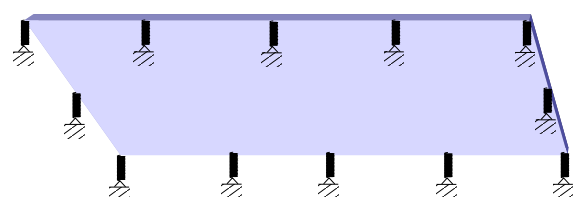

(c)

Figure 3: $(\alpha)$ Plan view of the sound laboratory setup used in ABAQUS model. (b) Depiction of the vibro-acoustic model. (c) Positioning of the twelve (12) mounts on the surface of the panel.

The panel is discretized by 20-node quadratic solid hexahedral elements, while the fluid domain is discretized by 20-node quadratic acoustic hexahedral elements. Tie constraints are used to simulate the coupling at the fluid-structure interface, while reflecting boundary conditions were specified to simulate the room conditions. In cases that the plate is supported on elastic mounts, connectors are installed on the required locations with the appropriate properties. Lastly, the discretization of the fluid domain has more than three quadratic elements across the wavelength of interest to increase the accuracy of the computational results [26].

\section{Numerical Example - Parametric investigation of STL}

In this section, the elements that affect STL of a rectangular plate are investigated, especially in case of a single leaf plasterboard partition. Initially, the analytic models proposed in the previous section A $0.625 \times 1.6 \mathrm{~m}$ rectangular plasterboard is considered, according to real-life masonry applications. The width corresponds to the horizontal distance between the upright supporting beams providing the stable frame of the plasterboard. Typically, the height of such a plasterboard is around $3.2 \mathrm{~m}$, meaning that it can be covered by two pieces with the dimensions consider here. The selected dimensions correspond also to a frontal surface of $1 \mathrm{~m}^{2}$. The relevant properties of the plasterboard are summarized in Table 1.

Table 1: Plasterboard properties.

\begin{tabular}{llllll}
\hline$\rho\left[\frac{\mathrm{kg}}{\mathrm{m}^{3}}\right]$ & $L_{x}[\mathrm{~m}]$ & $L_{y}[\mathrm{~m}]$ & $h[\mathrm{~m}]$ & $E[\mathrm{MPa}]$ & $\nu[-]$ \\
\hline 668 & 0.625 & 1.6 & $12.510^{-3}$ & 2900 & 0.31 \\
\hline
\end{tabular}

The red dashed line in Fig.4, represents the FFR panel approximation as given by Eq.(18). Intuitively, this approximation should constitute the ideal case regarding the STL of a panel 
with finite dimensions and may act as a reference curve for the various comparisons instead of the infinite panel approximation which is invalid in the lower frequency range that is being examined. The yellow line corresponds to the $1^{\text {st }}$ mode approximation of the simply supported panel while the blue dotted line is the lumped parameter model as derived from the $1^{\text {st }}$ mode approximation. The difference between the two methods is essentially the effect of fluid loading (FL), whereas in the latter model is not included. It is observed that the fluid loading has very little effect on the damping of the first resonance. Thus, the elimination of its participation in the respective models is justified and consists a safe approximation. Lastly, the black dashed line represents the modal superposition method as formulated by Roussos and the grey line with markers shows the STL as calculated from the finite element model. In general a good agreement is observed among all the applied methods.

\subsection{Mathematical vs Numerical models}

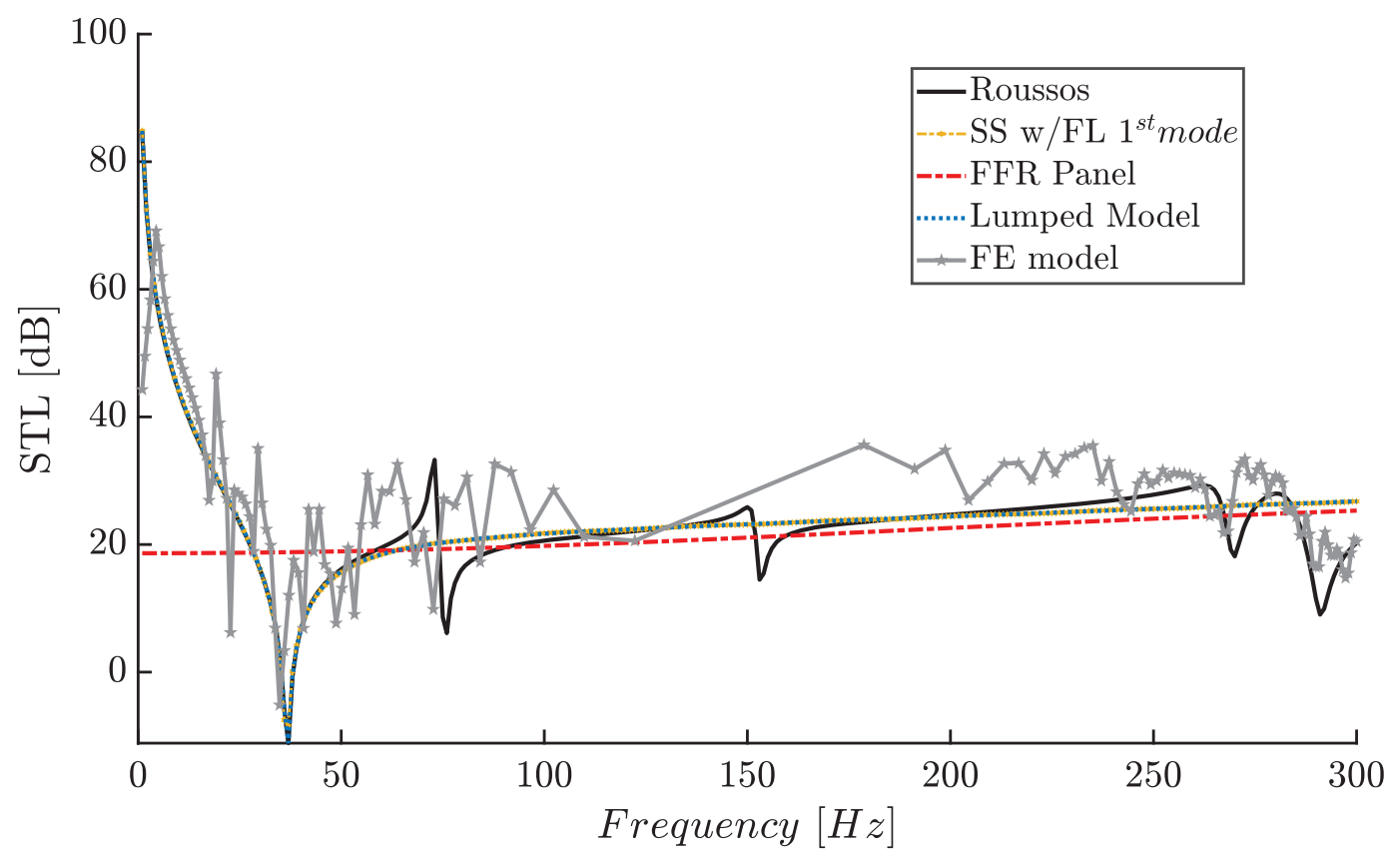

Figure 4: Comparison of $1^{\text {st }}$ mode, rigid approximations and numerical model.

The objective of the simplified models is to provide a more straightforward and fast prediction of the acoustic performance of the panel along with the ability to extract intuitive and revealing expressions of the main dynamic parameters that govern its frequency response. Since the higher modes have significantly lower modal participation factors in the frequency response than the fundamental mode, they are relatively easily damped. With that thinking, the approximation using only the $1_{s t}$ mode is considered appropriate in order to study this low frequency region, and following, the effect of elastic mounting and damping.

\subsection{Effect of support conditions in STL}

A case for the resulting STL frequency response compared to the simply-supported and clamped configurations are presented in Fig.5 $\alpha$. The total stiffness of the mounting is considered equal to the generalized stiffness of the simply supported panel $\left(k=k_{1}^{*}=1.110^{5} \mathrm{~N} / \mathrm{m}\right)$, 


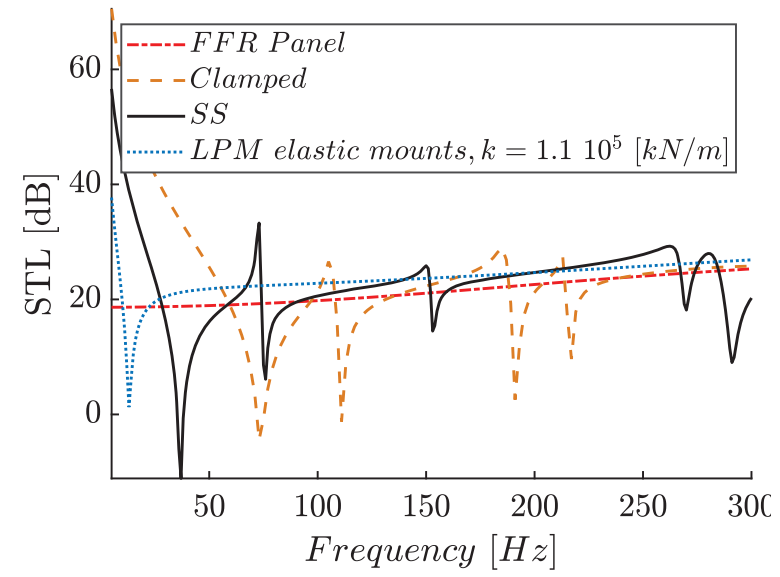

(a)

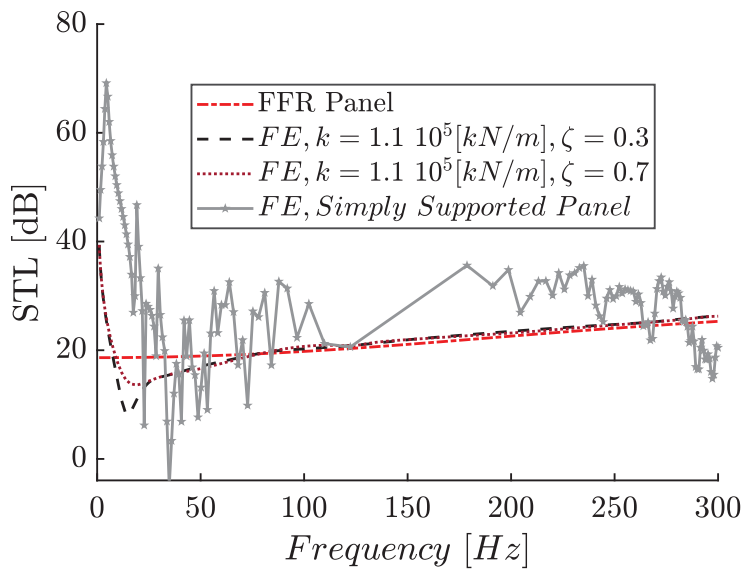

(b)

Figure 5: $(\alpha)$ Effect of support conditions in Sound transmission loss $(S T L)$. (b) Effect of damping in Sound transmission loss $(S T L)$.

while, no damping is considered for the mounts. This choice for the mounting stiffness is based on the Lamped parameter model, described in Section 2.5. Specifically, the total mounting stiffness is selected to drop the $1_{s t}$ resonance below $20[\mathrm{~Hz}]$ and then the estimated stiffness is allocated to the 12 springs of the FE model.

Following the logic of classic vibration isolation, by lowering this frequency enough it can be moved outside the frequency range of interest, e.g. above $20 \mathrm{~Hz}$. However, significantly decreasing this frequency can have certain implications. On one hand, it leads to slightly reduced STL frequency response and on other hand, it may present practical problems including excessive vibration magnitude, possible resonances with other supportive structural elements such as bolts or the frame, or even inadequate rigidity for impact loads. The optimal solution requires a combination that leads to a low enough fundamental eigenfrequency that can be effectively dampend without the need of excessive damping while at the same time is high enough that the overall STL is not significantly reduced and no vibrational implications come into play when the mounting is very soft.

Another purpose of the comparison in Fig. $5 \alpha$ is to highlight the deviation of the eigenfrequencies between the simply supported and clamped panels in regards to the uncertainty introduced by this fact in real world installations. Depending on the technique of the installation the exact support conditions may vary between these two cases. However, the inclusion of the soft elastic mounts, eliminates this uncertainty as it produces an explicit, calculated frequency response by design. Additionally, the reduction of the eigenfrequencies alone almost eliminates the participation of the higher modes even without the consideration of any energy diffusive elements besides the structural damping of the panel itself which holds for all three case compared here.

\subsection{Effect of damping}

Further examination considers the effect of damping of the elastic mounts. In order to investigate the effects of damping 12 linear springs were attached to the panel as in Fig.3c. Assuming the same stiffness for the mounts as in the previous case, a comparison is presented in Fig. $5 \mathrm{~b}$ damping ratios $\zeta=0.3$ and $\zeta=0.7$.

The results presented in Fig.5b are meant to demonstrate the expected level of damping in 
order to effectively damp the fundamental frequency. As already mentioned, even in cases of low damping the STL response is flattened as the higher eigenfrequencies are very effectively damped while only the first eigenfrequency requires significant damping. How realistic are the actual values of the assumed viscous dampers for high damping ratios is surely a matter of discussion and very much depends on practical factors like the material used for the mounts and spatial considerations of the real world implementation.

Certainly, the positions of the mounts have a significant role on their damping effectiveness. Especially in this case of viscous damping, the velocity amplitude is maximum at the center of the panel and minimum along the edges, therefore a mount positioned at the center would most effectively dampen the fundamental resonance. However, this case would change the dynamic behaviour of the panel and would also require an additional supporting frame along the middle of the panel for example, which is something that is preferably avoided. For this reasons such a modification is not investigated in the current contribution.

\section{Conclusions}

The variation of the support conditions of the panel, between simply supported and clamped, in a real-world implementation affects significantly the eigenfrequencies and therefore the frequency response of the STL.

The term "soft mounts" is approximately defined as the case in which the total stiffness of the mounts is lower than the generalized stiffness of the simply supported panel. When the panel is supported regionally on soft elastic mounts, the values of the eigenfrequencies of the structure are reduced. This has two main consequences:

- The value of the fundamental eigenfrequency can be explicitly defined, depending on the stiffnes of the mounts, and be moved outside the frequency range of interest, namely below $20 \mathrm{~Hz}$.

- The contribution of the higher modes is almost nullified without the need of any significant damping in the mounts.

Therefore, the utilization of soft mounts displaces the STL curve towards lower frequencies, providing smooth performance in the entire low frequency range. Additionally, this practice diminishes any uncertainties introduced in the acoustic performance either due to practical support conditions or variations in the rigidity of the panel.

Finally, the analytic models showed good agreement with the developed FE model, indicating that they can be used as a quick tool for the design of soft mounts.

\section{Acknowledgments}

Moris Kalderon has been financed by the European Union's Horizon 2020 research and innovation programme under the Marie Sklodowska-Curie grant (grant agreement No INSPIRE813424, ’INSPIRE - Innovative Ground Interface Concepts for Structure Protection”).

\section{Appendix}

\section{A.1 Far-field approximation}

The distance $R$ can be written as

$$
R=\sqrt{r^{2}-2 x \xi^{\prime}-2 y \eta^{\prime}+\xi^{\prime 2}+\eta^{\prime 2}}
$$


where $r=|\mathbf{r}|=\sqrt{x^{2}+y^{2}+z^{2}}$. In spherical coordinates $R$ becomes

$$
R=r \sqrt{1-2 \frac{\sin \theta}{r}\left(\xi^{\prime} \cos \phi+\eta^{\prime} \sin \phi\right)+\frac{\xi^{\prime 2}+\eta^{\prime 2}}{r}}
$$

The solution of Eq.(7) is not trivial and has been calculated analytically only for a few cases of boundary conditions. A way around this obstacle comes by utilizing the far field approximation. In the far field we can ignore the weak dependence of the amplitude of the integrand on the position but not the dependence of the phase, namely it holds that

$$
\begin{aligned}
\frac{1}{R} & \approx \frac{1}{r} \\
e^{-\jmath \kappa R} \approx e^{-\jmath \kappa r\left[1-\frac{\sin \theta}{r}\left(\xi^{\prime} \cos \phi+\eta^{\prime} \sin \phi\right)\right]} & =e^{-\jmath \kappa r} e^{\jmath \kappa \sin \theta\left(\xi^{\prime} \cos \phi+\eta^{\prime} \sin \phi\right)}
\end{aligned}
$$

Since $r$ is independent of $\xi^{\prime}$ and $\eta^{\prime}$, the corresponding terms in Eq.(7) can be moved outside the double integral, leading to

$$
p_{t}(\mathbf{r}, t)=\frac{\jmath \Omega \rho_{0}}{2 \pi r} e^{\jmath(\Omega t-\kappa r)} \int_{-l_{x} / 2}^{l_{x} / 2} \int_{-l_{y} / 2}^{l_{y} / 2} \frac{\partial w\left(\xi^{\prime}, \eta^{\prime}\right)}{\partial t} e^{\jmath \kappa\left(\xi^{\prime} \sin \theta \cos \phi+\eta^{\prime} \sin \theta \sin \phi\right)} d \eta^{\prime} d \xi^{\prime}
$$

which may be analytically calculated.

\section{A.2 Free Finite Rigid (FFR) Panel Approximation}

Then, the transmitted pressure by considering the far-field approximation, is calculated from the expression

$$
p_{t}(\mathbf{r}, t)=\frac{-\Omega^{2} \rho_{0} W}{2 \pi} \frac{e^{-\jmath k r}}{r} e^{\jmath \Omega t} \int_{-\frac{l_{x}}{2}}^{\frac{l_{x}}{2}} \int_{-\frac{l_{y}}{2}}^{\frac{l_{y}}{2}} e^{\jmath \kappa\left(\xi^{\prime} \sin \theta \cos \phi+\eta^{\prime} \sin \theta \sin \phi\right)} d \eta^{\prime} d \xi^{\prime}
$$

where the surface integral is calculated

$$
I_{\xi^{\prime} \eta^{\prime}}=\frac{8 \sin \left(\sigma_{x}\right) \sin \left(\sigma_{y}\right)}{k^{2} \sin ^{2} \theta \sin 2 \phi}
$$

and

$$
\begin{aligned}
\sigma_{x}(\theta, \phi) & =k \frac{l_{x}}{2} \sin \theta \cos \phi \\
\sigma_{y}(\theta, \phi) & =k \frac{l_{y}}{2} \sin \theta \sin \phi
\end{aligned}
$$

The transmitted intensity $I_{t}$ and consequently the transmitted power, are calculated from Eqs.(9),(10) where $I_{\theta \phi}$ is defined as

$$
I_{\theta \phi}=\int_{0}^{2 \pi} \int_{0}^{\pi / 2}\left|I_{\xi^{\prime} \eta^{\prime}}\right|^{2} \sin \theta d \theta d \phi
$$




\section{A.3 Simply Supported Plate - First Mode Approximation}

Considering only the $1^{\text {st }}$ mode for interpolation of the transverse displacement the derivatives to be substituted in the equation of motion are calculated as

$$
\begin{aligned}
\frac{\partial^{2} w(\xi, \eta)}{\partial t^{2}} & =-\Omega^{2} W_{1}(\xi, \eta) e^{-\jmath \Omega t} \\
\frac{\partial^{4} W_{1}(\xi, \eta)}{\partial \xi^{4}} & =C_{1}\left(\frac{\pi}{l_{x}}\right)^{4} \sin \left(\frac{\pi \xi}{l_{x}}\right) \sin \left(\frac{\pi \eta}{l_{y}}\right)=\left(\frac{\pi}{l_{x}}\right)^{4} W_{1}(\xi, \eta) \\
\frac{\partial^{4} W_{1}(\xi, \eta)}{\partial \eta^{4}} & =C_{1}\left(\frac{\pi}{l_{y}}\right)^{4} \sin \left(\frac{\pi \xi}{l_{x}}\right) \sin \left(\frac{\pi \eta}{l_{y}}\right)=\left(\frac{\pi}{l_{y}}\right)^{4} W_{1}(\xi, \eta) \\
\frac{\partial^{4} W_{1}(\xi, \eta)}{\partial \xi^{2} \partial \eta^{2}} & =C_{1}\left(\frac{\pi^{2}}{l_{x} l_{y}}\right)^{2} \sin \left(\frac{\pi \xi}{l_{x}}\right) \sin \left(\frac{\pi \eta}{l_{y}}\right)=\left(\frac{\pi^{2}}{l_{x} l_{y}}\right)^{2} W_{1}(\xi, \eta)
\end{aligned}
$$

Considering the far field approximation for the transmitted pressure and normal incident waves $\left(\theta_{i}=0\right)$, the equation of motion becomes

$$
\begin{array}{r}
{\left[D \pi^{4}\left(\frac{1}{l_{x}^{2}}+2 \frac{1}{l_{x}^{2} l_{y}^{2}}+\frac{1}{l_{y}^{2}}\right)-\Omega^{2} \rho h\right] W_{1}(\xi, \eta)=} \\
2 P_{i}+\frac{\Omega^{2} \rho_{0}}{\pi r} e^{-\jmath k r} I_{\xi^{\prime} \eta^{\prime}}
\end{array}
$$

where $I_{\xi^{\prime} \eta^{\prime}}$ is the double integral on the surface of the plate

$$
I_{\xi^{\prime} \eta^{\prime}}=\int_{-l_{x} / 2}^{l_{x} / 2} \int_{-l_{y} / 2}^{l_{y} / 2} W_{1}\left(\xi^{\prime}, \eta^{\prime}\right) e^{j k\left(\xi^{\prime} \sin \theta \cos \phi+\eta^{\prime} \sin \theta \sin \phi\right)} d \eta^{\prime} d \xi^{\prime}
$$

and the local coordinates $\xi^{\prime}=\xi-\frac{l_{x}}{2}, \eta^{\prime}=\eta-\frac{l_{y}}{2}$. For $\xi^{\prime}, \eta^{\prime}$ the shape function from Eq.(19) becomes

$$
W_{1}\left(\xi^{\prime}, \eta^{\prime}\right)=C_{1} \cos \left(\frac{\pi \xi^{\prime}}{l_{x}}\right) \cos \left(\frac{\pi \eta^{\prime}}{l_{y}}\right)
$$

The surface integral is then calculated as

$$
I_{\xi^{\prime} \eta^{\prime}}=C_{1} I_{\xi \eta}
$$

where

$$
I_{\xi \eta}=4 \pi^{2} l_{x} l_{y} \frac{\cos \left(\sigma_{x}\right)}{\left(l_{x} k \sin \theta \cos \phi\right)^{2}-\pi^{2}} \frac{\cos \left(\sigma_{y}\right)}{\left(l_{y} k \sin \theta \sin \phi\right)^{2}-\pi^{2}}
$$

and $\sigma_{x}, \sigma_{y}$ are the same as in Eq.(A.8). Solving (19) for $C_{1}$, the surface integral can be written as

$$
I_{\xi^{\prime} \eta^{\prime}}=\frac{W_{1}(\xi, \eta)}{\sin \left(\frac{\pi \xi}{l_{x}}\right) \sin \left(\frac{\pi \eta}{l_{y}}\right)} I_{\xi \eta}
$$


Substituting to equation (A.12), multiplying with $W_{1}$ and integrating over the panel surface gives

$$
\begin{array}{r}
\int_{0}^{l_{x}} \int_{0}^{l_{y}}\left[D \pi^{4}\left(\frac{1}{l_{x}^{2}}+\frac{1}{l_{y}^{2}}\right)^{2}-\frac{\Omega^{2} \rho_{0} e^{-\jmath \kappa r}}{\pi r} \frac{I_{\xi \eta}(\theta, \phi)}{\sin \left(\frac{\pi \xi}{l_{x}}\right) \sin \left(\frac{\pi \eta}{l_{y}}\right)}-\Omega^{2} \rho h\right] \ldots \\
\ldots W_{1}^{2}(\xi, \eta) d \eta d \xi=\int_{0}^{l_{x}} \int_{0}^{l_{y}} 2 P_{i} W_{1}(\xi, \eta) d \eta d \xi
\end{array}
$$

and inserting $\omega_{1}$ from Eq.(22) leads to

$$
\begin{aligned}
\rho h\left(\omega_{1}^{2}-\Omega^{2}\right) & \int_{0}^{l_{x}} \int_{0}^{l_{y}} W_{1}^{2}(\xi, \eta) d \eta d \xi= \\
& =\left[2 P_{i}+\frac{\Omega^{2} \rho_{0}}{\pi} \frac{e^{-\jmath \kappa r}}{r} C_{1} I_{\xi \eta}(\theta, \phi)\right] \int_{0}^{l_{x}} \int_{0}^{l_{y}} W_{1}(\xi, \eta) d \eta d \xi
\end{aligned}
$$

The two integrals are calculated as

$$
\begin{aligned}
& \int_{0}^{l_{x}} \int_{0}^{l_{y}} W_{1}^{2}(\xi, \eta) d \eta d \xi=\frac{l_{x} l_{y}}{4} C_{1}^{2} \\
& \int_{0}^{l_{x}} \int_{0}^{l_{y}} W_{1}(\xi, \eta) d \eta d \xi=\frac{4 l_{x} l_{y}}{\pi^{2}} C_{1}
\end{aligned}
$$

Substituting in Eq.(A.19) and solving the quadratic equation for $C_{1}$ leads to:

$$
C_{1}=\frac{8 P_{i}}{\pi^{2}\left[\rho_{0} h \frac{\left(\omega_{1}^{2}-\Omega^{2}\right)}{4}-\frac{4 \Omega^{2} \rho_{0}}{\pi^{3}} \frac{e^{-\jmath k r}}{r} I_{\xi \eta}(\theta, \phi)\right]}=\frac{32 P_{i}+p_{t}}{\pi^{2} \rho h\left(\omega_{1}^{2}-\Omega^{2}\right)}
$$

Therefore, substituting in Eq.(A.5) for the transmitted pressure, comes:

$$
\begin{aligned}
p_{t}(\mathbf{r}, t) & =\frac{\Omega^{2} \rho_{0} e^{-\jmath k r}}{2 \pi r} C_{1} I_{\xi \eta}(\theta, \phi) e^{\jmath \Omega t} \\
\Rightarrow p_{t}(\mathbf{r}, t) & =\frac{16 \Omega^{2} \rho_{0} I_{\xi \eta}(\theta, \phi) P_{i}}{r\left[\pi^{3} \rho h\left(\omega_{1}^{2}-\Omega^{2}\right)-16 \Omega^{2} \rho_{0} \frac{e^{-\jmath k r}}{r}\right]} e^{-\jmath k r} e^{\jmath \Omega t}
\end{aligned}
$$

The transmitted intensity $I_{t}$ is calculated from Eq.(9) as

$$
I_{t}(r, \theta, \phi)=\frac{1}{2 \rho_{0} c_{0} r^{2}}\left(\frac{16 \omega^{2}\left|I_{\xi \eta}\right| P_{i}}{\left|\pi^{3} \rho h\left(\omega_{1}^{2}-\Omega^{2}\right)-16 \Omega^{2} \rho_{0} \frac{e^{-\jmath \kappa r}}{r}\right|}\right)^{2}
$$

and the transmitted power comes from Eq.(10) as

$$
\Pi_{t}=\frac{P_{i}^{2}}{2 \rho_{0} c_{0}} \frac{\left(16 \Omega^{2} \rho_{0}\right)^{2}}{\left|\pi^{3} \rho h\left(\omega_{1}^{2}-\Omega^{2}\right)-16 \Omega^{2} \rho_{0} \frac{e^{-\jmath \kappa r}}{r}\right|^{2}} I_{\theta \phi}
$$

where $I_{\theta \phi}$ is the double integral over a hemispheric surface in the receiver domain

$$
I_{\theta \phi}=\int_{0}^{2 \pi} \int_{0}^{\pi / 2}\left|I_{\xi \eta}\right|^{2} \sin \theta d \theta d \phi
$$

and $I_{\xi \eta}$ is defined in Eq.(A.16). 


\section{A.3.1 Lumped Parameter Model}

Let us rewrite the transverse displacement as

$$
w(\xi, \eta, t)=\sin \left(\frac{\pi \xi}{l_{x}}\right) \sin \left(\frac{\pi \eta}{l_{y}}\right) q(t)
$$

where

$$
q(t)=C_{1} e^{\jmath \Omega t}
$$

The kinetic energy of the system is defined as

$$
\begin{aligned}
T & =\frac{1}{2} \rho h \int_{0}^{l_{x}} \int_{0}^{l_{y}}\left(\frac{\partial w}{\partial t}\right)^{2} d \xi d \eta \\
& =\frac{1}{2} \frac{\rho h l_{x} l_{y}}{4} \dot{q}^{2}(t) \\
& =\frac{1}{2} m_{1}^{*} \dot{q}^{2}(t)
\end{aligned}
$$

and the potential energy as

$$
\begin{aligned}
U & =\frac{1}{2} D \int_{0}^{l_{x}} \int_{0}^{l_{y}}\left[\nabla^{2} w(\xi, \eta, t)\right]^{2} d \xi d \eta \\
& =\frac{1}{2} \frac{D \pi^{4} l_{x} l_{y}}{4}\left(\frac{1}{l_{x}^{2}}+\frac{1}{l_{y}^{2}}\right)^{2} q^{2}(t) \\
& =\frac{1}{2} k_{1}^{*} q^{2}(t)
\end{aligned}
$$

where $m_{1}^{*}$ and $k_{1}^{*}$ are the generalized mass and stiffness of the fundamental, $1^{\text {st }}$ mode.

The equation of motion of the equivalent SDOF model is

$$
m_{1}^{*} \ddot{q}+k_{1}^{*} q=f^{*}(t)
$$

where the generalized excitation, considering only the blocked pressure, comes as

$$
f^{*}(t)=2 p_{i} \int_{0}^{l_{x}} \int_{0}^{l_{y}} W_{1}(\xi, \eta) d \xi d \eta=\frac{8 P_{i} l_{x} l_{y}}{\pi^{2}} e^{\jmath \Omega t}
$$

Consequently, by substituting in Eq.(A.31), and since $k_{1}^{*}=m_{1}^{*} \omega_{1}^{2}$, the factor $C_{1}$ is calculated as:

$$
C_{1}=\frac{8 P_{i} l_{x} l_{y}}{\pi^{2}} \frac{1}{m_{1}^{*}\left(\omega_{i}^{2}-\Omega^{2}\right)}
$$

\section{REFERENCES}

[1] Vigran E T. Building Acoustics. Taylor \& Francis Limited, 2019, 2019.

[2] Norén-Cosgriff K, Løvholt F, Brekke A et al. Countermeasures against noise and vibrations in lightweight wooden buildings caused by outdoor sources with strong low frequency components. Noise Control Engineering Journal 2016; 64: 737-752. 
[3] Løvholt F, Madshus C and Norén-Cosgriff K. Analysis of low frequency sound and sound induced vibration in a norwegian wooden building. Noise Control Engineering Journal 2011; 59(4): 383-396.

[4] ISO 717, acoustics - rating of sound insulation in buildings and of building elements. Standard.

[5] ISO 10140, acoustics — laboratory measurement of sound insulation of building elements. Standard.

[6] ISO 15186, acoustics - measurement of sound insulation in buildings and of building elements using sound intensity. Standard.

[7] Ayr U, Martellotta F and Rospi G. A method for the low frequency qualification of reverberation test rooms using a validated finite element model. Applied Acoustics 2017; 116: 33-42. DOI:https://doi.org/10.1016/j.apacoust.2016.09.001. URL https://www.sciencedirect.com/science/article/pii/s0003682X16302663.

[8] Løvholt F, Norèn-Cosgriff K, Madshus C et al. Simulating low frequency sound transmission through walls and windows by a two-way coupled fluid structure interaction model. Journal of Sound and Vibration 2017; 396: 203 - 216. DOI:https://doi.org/10.1016/j.jsv. 2017.02.026.

[9] Aretz $\mathrm{M}$ and Vorländer M. Efficient modelling of absorbing boundaries in room acoustic fe simulations. Acta Acustica United With Acustica 2010; 96: 1042-1050.

[10] Vorländer M. Computer simulations in room acoustics: Concepts and uncertainties. The Journal of the Acoustical Society of America 2013; 133(3): 1203-1213. DOI:10.1121/1. 4788978.

[11] Beranek LL and Vér IL. Noise and vibration control engineering: Principles and applications. John Wiley \& Sons, New York.

[12] Cremer L, Heckl M and Petersson BA. Structure-borne sound: structural vibrations and sound radiation at audio frequencies. Springer Science \& Business Media, 2005.

[13] Ordubadi A and Lyon RH. Effect of orthotropy on the sound transmission through plywood panels. The Journal of the Acoustical Society of America 1979; 65(1): 133-139. DOI:10.1121/1.382255.

[14] London A. Transmission of reverberant sound through double walls. The Journal of the Acoustical Society of America 1950; 22(2): 270-279. DOI:10.1121/1.1906601.

[15] Callister J, George A and Freeman G. An empyrical scheme to predict the sound transmission loss of single-thickness panels. Journal of Sound and Vibration 1999; 222(1): 145 151. DOI:https://doi.org/10.1006/jsvi.1998.1624.

[16] Pellicier A and Trompette N. A review of analytical methods, based on the wave approach, to compute partitions transmission loss. Applied Acoustics 2007; 68(10): $1192-1212$. DOI:https://doi.org/10.1016/j.apacoust.2006.06.010.

[17] Kim YH. Sound propagation: an impedance based approach. John Wiley \& Sons, 2010. 
[18] Paradeisiotis A, Kalderon M, Antoniadis I et al. Acoustic Performance Evaluation of a panel utilizing negative stifffness mounting for low frequency noise control. In Proceedings of EURODYN 2020. Athens, Greece, 23-26 November: EASD Procedia, pp. 4093-4110.

[19] Cowan AJ. Sound Transmission Loss of Composite Sandwich Panels. PhD Thesis, Department of Mechanical Engineering, University of Canterbury, 2013.

[20] Rayleigh JWSB. The Theory of Sound, volume 1. Macmillan, 1894, 1896. DOI:10.1017/ CBO9781139058087.

[21] Roussos L. Noise transmission loss of a rectangular plate in an infinite baffle. The Journal of the Acoustical Society of America 1985; 75. DOI:10.1121/1.2021367.

[22] Yang Y, Lam NTK and Zhang L. Estimation of response of plate structure subject to low velocity impact by a solid object. International Journal of Structural Stability and Dynamics 2012; 12(06): 1250053.

[23] Koblar D and Boltežar M. Evaluation of the frequency-dependent young's modulus and damping factor of rubber from experiment and their implementation in a finite-element analysis. Experimental Techniques 2013; .

[24] Carfagni M, Lenzi E and Pierini M. The loss factor as a measure of mechanical damping. In Proc. SPIE, Proceedings of the 16th International Modal Analysis Conference, volume 3243. p. 580.

[25] Smith M. ABAQUS/Standard User's Manual, Version 6.9. United States: Dassault Systèmes Simulia Corp, 2009.

[26] Marburg S. Six boundary elements per wavelength: Is that enough? Journal of Computational Acoustics 2002; 10(01): 25-51. DOI:10.1142/S0218396X02001401. 